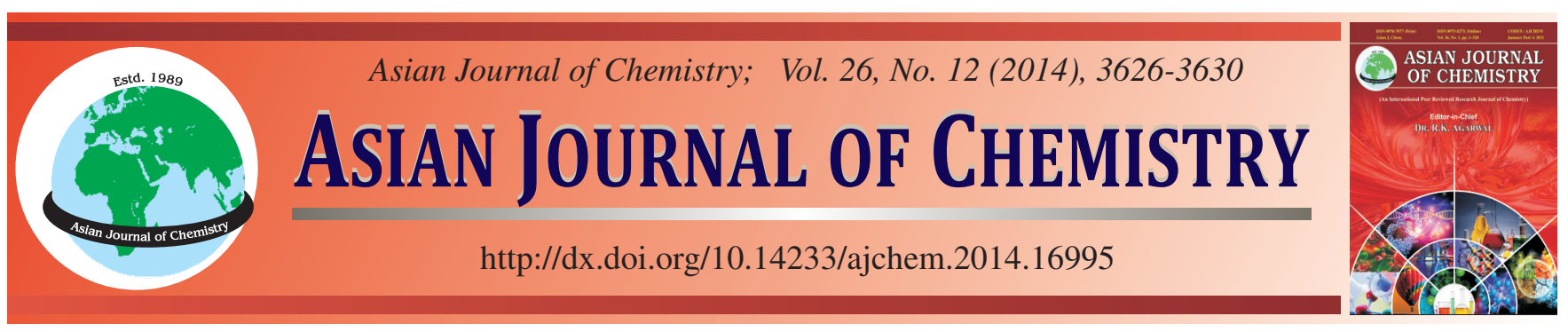

\title{
A Novel Deep Eutectic Solvents Synthesized by Solid Organic Compounds and Its Application on Dissolution for Cellulose
}

ERPENG ZHOU and Huiru LiU*

College of Chemical and Engineering, Shijiazhuang University, Shijiazhuang, P.R. China

*Corresponding author: Tel: +86 311 66617320; Fax: +86 311 66617326; E-mail: huiruliu16@163.com

\begin{abstract}
This study developed a new area of novel molten salt prepared by solid organic compounds, named deep eutectic solvents. Three deep eutectic solvents were synthesized by urea/caprolactam, caprolactam/acetamide and urea/acetamide at the molar ratio of 1:3, 1:1 and 1:2, respectively. The physical characters such as melt point, conductivity and solubility were investigated. It was shown that they were significantly affected by the mole ratio of the organic compounds and three systems at 1:3, 1:2 and 1:1 gave a liquid with the freezing point of 30,48 and $18^{\circ} \mathrm{C}$, respectively, which were much lower than those of raw materials. The conductivity in the range $10^{-5}$ to $10^{-4} \mathrm{~S} / \mathrm{m}$ changed with the temperature and accorded to Arrhenius equation. IR showed that the H-bondings of raw materials were disappeared and new H-bondings were formed. The application of three deep eutectic solvents on the dissolution for cellulose was investigated. It was found that the solubility of cellulose was low. The dissolving process for cellulose was the competition of deep eutectic solvents with the $\mathrm{H}$ bonding in cellulose. New H-bonding was formed between the hydroxy of cellulose and the oxygen of carbonyl and/or the nitrogen of amino group.
\end{abstract}

Keywords: Deep eutectic solvents, Cellulose, Ionic liquid, Dissolution.

\section{INTRODUCTION}

Chemistry is dominated by the study of species in solution. Although any liquid may be used as a solvent, relatively few are in general use. However, as the introduction of cleaner technologies has become a major concern throughout both industry and academia, the search for "Green solvent" has become a high priority. It has been proposed that ionic liquids (ILs) provide a useful extension to the range of solvents that are available for chemistry. Their negligible vapor pressure, excellent thermal stabilities and their polar and non-coordinating properties, especially the provision of a simple and effective method of recovering products and recycling catalysts have made them preferable solvents for green chemistry ${ }^{1-9}$. Furthermore, they can enhance the rate and increase the selectivity of a number of valuable reactions ${ }^{10}$. However, ionic liquids is difficult to be purified and often contain impurities which limit their application to some extent ${ }^{1}$. At present, many studies have been devoted to improve ionic liquids types and their applications. Eutectic mixtures of salts which are similar with ionic liquids have been extensively utilized to decrease the temperature for molten salt applications. Ambient temperature molten salts have been formed by mixing quaternary ammonium salts with metal salts ${ }^{11-15}$. This type of ionic liquids can be viewed as a deep eutectic resulting from the formation of complex anions, e.g., $\mathrm{Al}_{2} \mathrm{Cl}_{7}{ }^{-}$and $\mathrm{Zn}_{2} \mathrm{Cl}_{5}{ }^{-}$. These ideas have recently been extended to other chlorometalate salts including those formed from $\mathrm{ZnCl}_{2}, \mathrm{SnCl}_{2}$ and $\mathrm{FeCl}_{3}{ }^{16}$. Recently, studies have centered on imidazolium based cations with a range of anions including $\mathrm{BF}_{4}^{-}, \mathrm{PF}_{6}^{-}$and $\left(\mathrm{CF}_{3} \mathrm{SO}_{2}\right)_{2} \mathrm{~N}^{-}$. While these solvents show excellent solubility for a wide range of solutes and unusual miscibility with molecular liquids, their widespread application will be limited by toxicological, economic and purity issues. Base on the idea, a eutectic mixture prepared by a variety of quaternary ammonium salts and carboxylic acids gave a liquid ${ }^{17}$. This liquid was found to have interesting solvent properties that are similar to ambient temperature ionic liquids and named deep eutectic solvent (DES). Deep eutectic solvent was another green solvent that are similar to ambient temperature ionic liquids ${ }^{17,18}$. Some simple physical properties that make them interesting as potential solvents for chemistry are the following: (1) They are stable at wide liquid region. (2) A wide variety of solutes were found to exhibit high solubilities. (3) they are nonvolatile, hence they may be used in high-vacuum systems and eliminate many containment problems.

Cellulose is one of the most abundant bio-renewable materials, with a long and well-established technological base ${ }^{19,20}$. Products derived from cellulose have many important 
applications in fiber, paper, film, polymer and paints industries. However, natural cellulose is high crystalloid, with strong interand intra-molecular $\mathrm{H}$ bonding caused by the hydroxyl groups and is insoluble in water and common organic solvents. The searching for "Green solvents" on cellulose has become a high priority. Swatloski et al. ${ }^{20}$ led the first research on developing uses of ionic liquids as cellulose solvents. They reported in 2002 that the ionic liquid 1-butyl-3-methyl imidazole chloride $\left(\left[\mathrm{C}_{4} \mathrm{mim}\right] \mathrm{Cl}\right)$ exhibited super solubility for cellulose. Recently, more than 20 ionic liquids have shown promise as a class of new solvents which can dissolve cellulose. Cationic types of ionic liquids which have been studied include: alkyl quaternary ammonium cation $\left[\mathrm{NR}_{\mathrm{x}} \mathrm{H}_{4-\mathrm{x}}\right]^{+}$, alkyl quaternary phosphonium cation $\left[\mathrm{RR}_{\mathrm{x}} \mathrm{H}_{4-\mathrm{x}}\right]^{+}, \mathrm{N}$-alkyl pyridine cation $\left[\mathrm{RP}_{\mathrm{y}}\right]^{+}$and N,N-2alkyl imidazole cation $\left[\mathrm{R}_{1} \mathrm{R}_{3} \mathrm{im}\right]^{+}$, where "im" represents the imidazole structure ${ }^{21}$. To our best of knowledge, deep eutectic solvent as a solvent for cellulose has not been reported. In the current work, we focus on a novel kind of eutectic solvents that is synthesized by two organic solids compounds, such as caprolactam, acetamide and urea. These eutectic mixtures are easy to prepare in a pure state. The first application as solvent for cellulose is also investigated. This research not only extends the field of ionic liquids and deep eutectic solvent but also provides a new approach for the researching of solvent on cellulose.

\section{EXPERIMENTAL}

Caprolactam, urea and acetamide were purchased from China of Tianjin Chemical Reagent Factory and further dried under vacuum prior to use. The melt point of three reagents is $68-70,133$ and $78-81{ }^{\circ} \mathrm{C}$, respectively. Alcohol was used as received.

Cotton-ramie pulp obtained from commercial sources was used as the cellulose sample, with a degree of polymerization (DP) of 517. All cotton-ramie pulps were cut into small pieces and dried at $100{ }^{\circ} \mathrm{C}$ for $12 \mathrm{~h}$ without activation treatment before use.

Preparation of deep eutectic solvent: The urea and caprolactam were added to the beaker at different molar ratio. The mixture was placed on heating jacket and stirred until a homogeneous colorless liquid was formed. All the operation was processing in glove box with $\mathrm{N}_{2}$. After that, the compound was dried in vacuum at $25^{\circ} \mathrm{C}$. The final compound was sealed at vessel. The procedures of preparing caprolactam/acetamide and acetamide/urea deep eutectic solvent were same with above. The obtained liquids were cooled at a rate of $1{ }^{\circ} \mathrm{C} \mathrm{min}-1$ and the freezing point was taken as the temperature at which the first solid began to form.

Dissolution of cellulose: The dissolution of cellulose was processed using Zhao et al. ${ }^{22}$ and Liu et $a l_{.}{ }^{23}$ as reference. In the dissolving process, a certain amount of cellulose sample was added into the ionic liquids. The flask was continuously purged with gaseous $\mathrm{N}_{2}$ and the cellulose solution was stirred continuously in an oil bath at $120^{\circ} \mathrm{C}$. Because of high crystallinity of cellulose, the process of dissolution of cellulose was monitored with a polarizing optical microscope. After the emergence of a blackburst in the cellulose solution, as determined on a polarization microscope, the next level of cellulose sample was added into the dissolved cellulose solution. The above experimental procedure was repeated for several cyclic numbers. The solubility of cellulose corresponding to dissolution times in ionic liquids for each cyclic number was recorded.

Detection method: FTIR spectra of the synthesized deep eutectic solvent were recorded on a FTS-135 spectrometer (BIO-RAD, America) using the $\mathrm{KBr}$ wafer technique. The conductivity and its temperature dependence were determined using a DDS-II conductivity meter with temperature and conductivity probes (probe cell constant $1 \mathrm{~cm}^{-1}$ ). The dissolution process of cellulose was monitored by using a polarizing microscope (XP-203, Shanghai Changfang Optical Instrument Co., Ltd., China).

\section{RESULTS AND DISCUSSION}

Melting point: Melting point is a key parameter to evaluate deep eutectic solvent. The melting point depended on the molar ratio of two compounds. With the molar ratio increasing or decreasing to the optimal value, the melting point increase. The optimal molar ratio of different deep eutectic solvent and the corresponding melting point were showed in Table-1. For every compound, it could be seen that the melting point was much lower than that of the raw materials. It was evidence that caprolactam, urea and acetamide combine of two and produce new specie. In addition, according to the definition of ionic liquid, it was present liquid at wide range of molar ratio of two materials, but a lowest melt point at optimal ratio. We deduced that two organic molecule combined by $\mathrm{H}-$ bonding and formed super-molecule. Organic solid compounds containing $\mathrm{H}$-bonding are polar molecule, which is formed by regularly arranging of $\mathrm{H}$-bonding. For the simple discussion, deep eutectic solvent of caprolactam/urea was chosen as an example. When the mixing-system heated, the intramolecular $\mathrm{H}$-bonding of each precursor compound was destroyed and new intermolecular H-bondings between a urea molecule and more caprolactam molecules were formed, which led to the decrease of some physical factors, such as energy of H-bonding, the degree of molecular arrange and the electrostatic attraction between hydrogen and organic molecule. Generally, low symmetry, weak ion interactions (such as suppressing $\mathrm{H}$ bonding) and effective charge distribution over the cation and/or anion tends to reduce the crystal lattice energy of the salts, thus resulting in low-melting salts ${ }^{24}$. Theoretically, two organic materials with H-bonding can synthesize this type of deep eutectic solvent. It must be noted that in this type deep eutectic solvent, only the solid organic materials such as solid alcohol, sugar, solid aldehyde, etc., were considered.

\begin{tabular}{cccc}
\multicolumn{4}{c}{ TABLE-1 } \\
& $\begin{array}{c}\text { MELTING POINT OF SYNTHESIZED } \\
\text { DEEP EUTECTIC SOLVENT }\end{array}$ \\
\hline \multirow{2}{*}{ Systems } & Urea/ & Caprolactam/ & Urea/ \\
& Caprolactam & Acetamide & Acetamide \\
\hline Optimal mole ratio & $1: 3$ & $1: 1$ & $1: 2$ \\
Melting point $\left({ }^{\circ} \mathrm{C}\right)$ & 30 & 18 & 48 \\
\hline
\end{tabular}

Conductivity: The conductivity of synthesized deep eutectic solvent at optimal molar ratio was about $10^{-5}-10^{-4} \mathrm{~S} / \mathrm{m}$. Compared with ionic liquids, the conductivity of deep eutectic 
solvent was very low, especially that of deep eutectic solvent synthesized by urea and caprolactam, which was only about $3.2 \times 10^{-5} \mathrm{~S} / \mathrm{m}$ at $38^{\circ} \mathrm{C}$. At the same temperature, the conductivity of three systems was following: Urea/caprolactam < caprolactam/acetamide < urea/acetamide. At present, the key property that distinguishes deep eutectic solvent from ionic liquids is the low conductivity. The change of conductivity with temperature was shown in Figs. 1 and 2. With the increasing of temperature, the conductivity increased. The temperature and conductivity was accorded to Arrhenius equation.

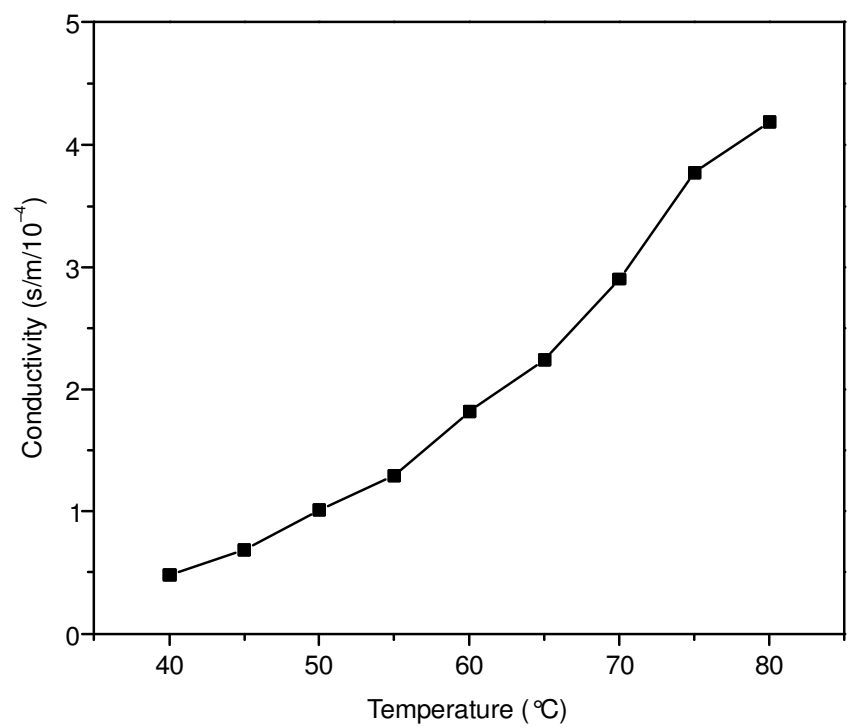

Fig. 1. Conductivity profiles of urea/caprolactam deep eutectic solvent with temperature

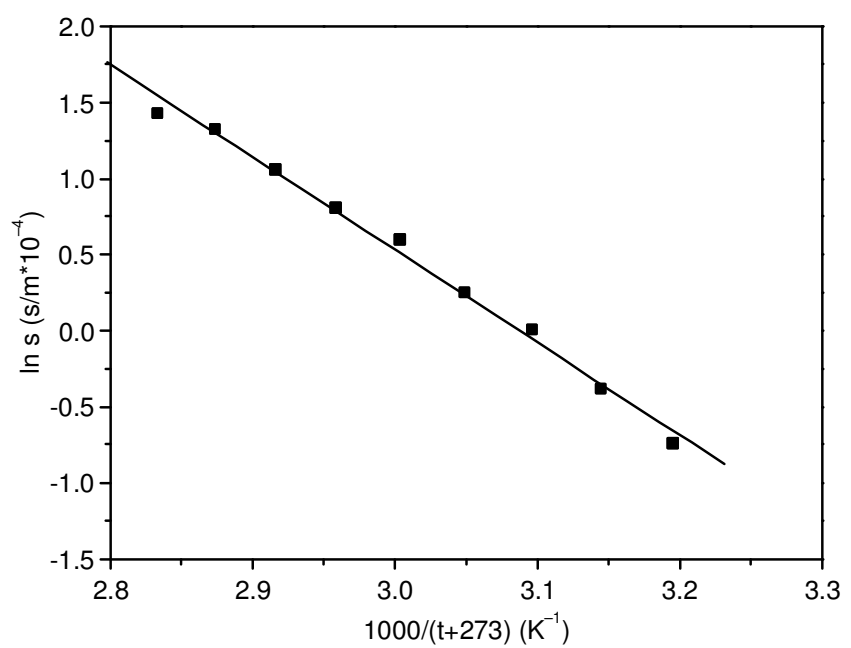

Fig. 2. Arrhenius curve of conductivity of urea/caprolactam deep eutectic solvent with temperature

Conductivity is the ability of material transferring ion. So the number of mobile ions influences the conductivity. For three kinds of deep eutectic solvent studied in this work, the low conductivity showed that there were nearly few ions existing in the synthesized system. As discussed above, two organic compounds were formed by regularly arranging of $\mathrm{H}$-bonding. In the processing of synthesis, the two organic compounds may be combined by the $\mathrm{H}$-bond and formed a three-dimensional H-bonding network, namely the super- molecule. For these systems, the freely moving particle was not the single molecule, but the super-molecule and/or free molecules. The moving of super-molecules could form larger super-molecules, while larger super-molecules can also decompose to small super-molecules. Thus, it can be deduced that the low conductivity was not produced by ion, but the moving of super-molecules and free molecules.

IR spectra: Figs. 3-5 showed the IR spectra of urea, caprolactam and systhesized deep eutectic solvent, respectively. In Fig. 3, absorption spectra of 2920.4, 1395 and $1671.4 \mathrm{~cm}^{-1}$ were the $\mathrm{C}-\mathrm{H}$ vibration, $\mathrm{C}-\mathrm{N}$ stretching vibration and $\mathrm{C}=\mathrm{O}$ vibration of caprolactam, respectively. The $\mathrm{N}-\mathrm{H}$ vibration of urea in Fig. 4 appeared in absorption spectra of 3454.5 and $1617.2 \mathrm{~cm}^{-1}$. Comparing the FTIR spectra of deep eutectic solvent with the urea and caprolactam, the N-H vibration of urea disappeared and $\mathrm{C}=\mathrm{O}$ vibration of caprolactam was shifted from 1671.4 to $1649.4 \mathrm{~cm}^{-1}$. From all the information, it can be deduced that $\mathrm{C}=\mathrm{O}$ of caprolactam and hydrogen in urea can be connected $(\mathrm{C}=\mathrm{O}-\mathrm{H}-\mathrm{N})$.

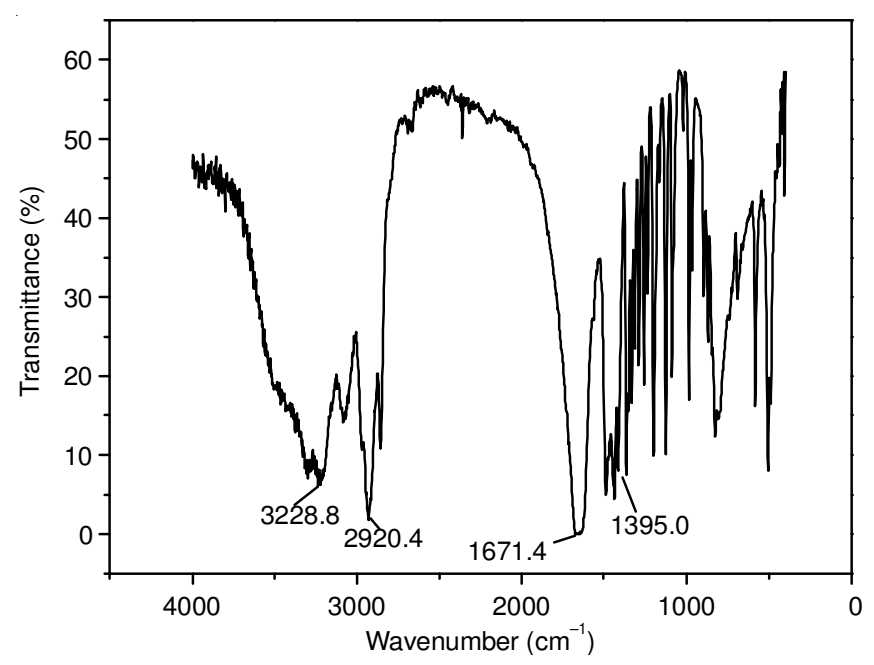

Fig. 3. IR spectra of caprolactam

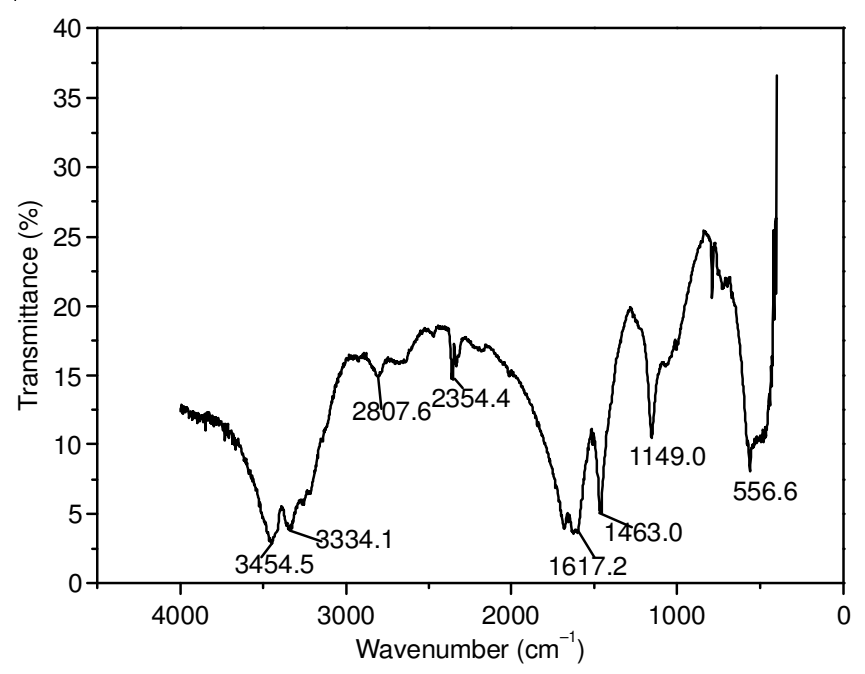

Fig. 4. IR spectra of urea

Solubility of cellulose: The solubility of cellulose in different deep eutectic solvent was summarized in Table-2. As seen in Table-2, the three solvent can dissolve cellulose 
under the order: urea/caprolactam > caprolactam/acetamide > urea/acetamide, but the dissolvability of ionic liquids was low. In this type deep eutectic solvent, the dissolving process for cellulose was the competition of deep eutectic solvent with the $\mathrm{H}$ bonding in cellulose. For the deep eutectic solvent, expect for the intra- $\mathrm{H}$ bonding, there were also the inter- $\mathrm{H}$ bonding formed by cellulose and deep eutectic solvent. Therefore, it was favorable to understanding the dissolving process of cellulose by analyzing the ability of forming $\mathrm{H}$ - bonding and the type of H-bonding. Fig. 6 illustrated the type and the amount of hydrogen bond on the three deep eutectic solvent. One amino can form three $\mathrm{H}$-bondings (two hydrogen atoms of nitrogen and the ione pair electron of nitrogen) and oxygen of carbonyl can form two H-bondings. For one urea molecule, there are one carbonyl and two amines, which can form eight H-bondings. For the same analyzing, one caprolactam molecule and one acetamide molecule can form four H-bondings and five H-bondings, respectively. From the Fig. 6, it can be seen there were three, three and five H-bondings, in three deep eutectic solvent of urea/caprolactam, caprolactam/acetamide and urea/acetamide, respectively. Through the above analysis, it can be deduced that the three deep eutectic solvent can form five, three and three H-bonding with cellulose. For the system of cellulose-DES of caprolactam/acetamide and cellulose-DES of urea/acetamide, the hydrogen bond energy of oxygen on the carbonyl group was higher than that of nitrogen on the amido, so the solubility of caprolactam/acetamide was higher than that of urea/acetamide. As discussed above, the solubility of deep eutectic solvent for cellulose depended on the amount and intensity of H-bonding formed with cellulose. Namely, the more hydrogen bond formed with cellulose was, the higher solubility of cellulose was.

\begin{tabular}{cccc} 
TABLE-2 & \multicolumn{3}{c}{ SOLUBLITY OF CELLULOSE IN DEEP EUTECTIC SOLVENTS } \\
\hline \multicolumn{3}{c}{ SOLUbility of cellulose/g } \\
\hline $\begin{array}{c}\text { Dissolution } \\
\text { temperature } \\
\left({ }^{\circ} \mathrm{C}\right)\end{array}$ & $\begin{array}{c}\text { Urea/caprolactam } \\
(1: 3)\end{array}$ & $\begin{array}{c}\text { Caprolactam/ } \\
\text { acetamide } \\
(1: 1)\end{array}$ & $\begin{array}{c}\text { Urea/acetamide } \\
(1: 2)\end{array}$ \\
\hline 50 & 2.83 & 1.79 & 1.03
\end{tabular}

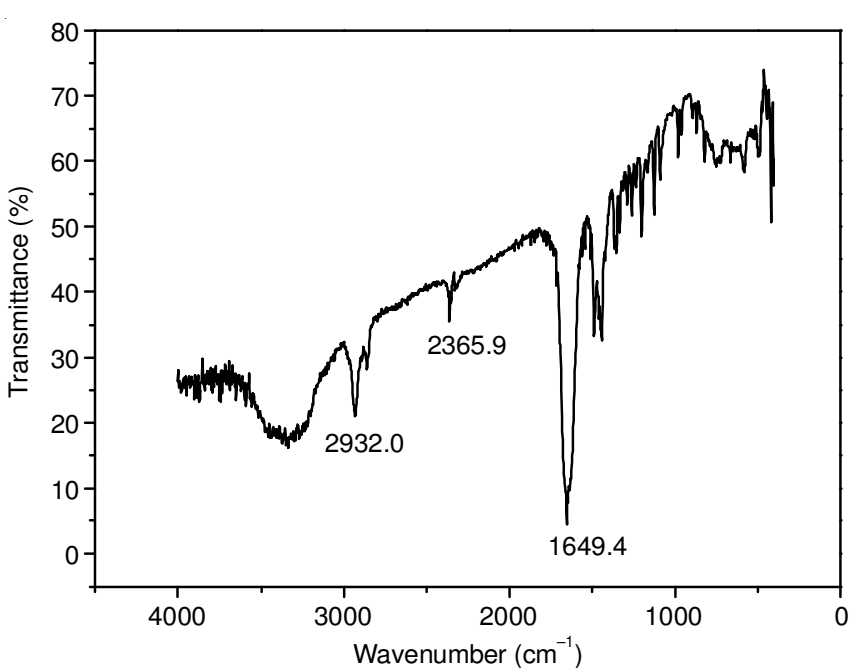

Fig. 5. IR spectra of deep eutectic solvent synthesized by urea and caprolactam at mole ratio of $1: 3$

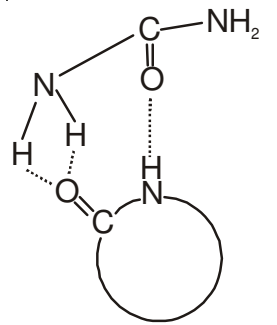

A. Urea/caprolactam

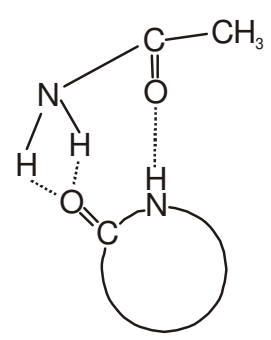

B. Caprolactam/acetamide

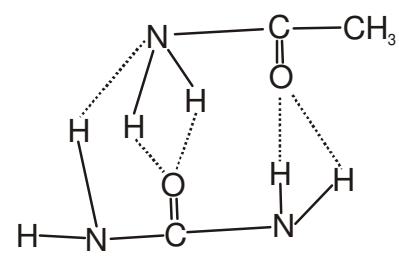

C. Urea/acetamide

Fig. 6. Intermolecular hydrogen bonding of deep eutectic solvent

The H-bonding can be formed between hydroxyl group of cellulose and oxygen on carbonyl group or/and nitrogen on amino group of this type deep eutectic solvent, which were amide solvent. The dissolvability of cellulose depended on the competitive formation of hydrogen bond between amide and inter-cellulose, which was formed through hydroxyl group on $\mathrm{C}_{3}$ and $\mathrm{C}_{6}$. The low solubility in Table- 2 demonstrated that the hydrogen bond energy between amide and hydroxyl group was same or stronger than that of inter cellulose. In order to increase the solubility of cellulose, the solvent that could form stronger hydrogen bond with cellulose need to be prepared.

\section{Conclusion}

A novel kind of eutectic solvents, namely deep eutectic solvent is synthesized by urea/caprolactam, caprolactam/ acetamide and urea/acetamide. Tow compound can be combined with H-bondings. Three deep eutectic solvent has low melt point and low conductivity which was not produced by ion, but the moving of super-molecules and free molecules. Theoretically, this type of deep eutectic solvent can be synthesized by two organic materials with H-bonding such as solid alcohol, sugar, solid aldehyde, etc.

This type of deep eutectic solvent is amide solvent. The dissolving process was the formation of new H-bonding between hydroxyl group of cellulose and oxygen on carbonyl group or/and nitrogen on amino group of this type deep eutectic solvent. The dissolvability of cellulose depends on the competitive formation of hydrogen bond between amide and intercellulose, which was formed through hydroxyl group on $\mathrm{C}_{3}$ and $\mathrm{C}_{6}$.

\section{ACKNOWLEDGEMENTS}

This work has been supported by the Natural Science Foundation of China under contract number 20976107. The work has been also supported by the HeBei Science Foundation and Doctor Scientific Research Foundation of Shijiazhuang University. 


\section{REFERENCES}

1. T. Welton, Chem. Rev., 99, 2071 (1999).

2. J. Dupont, R.F. de Souza and P.A.Z. Suarez, Chem. Rev., 102, 3667 (2002).

3. C. Baudequin, J. Baudoux, J. Levillain, D. Cahard, A.-C. Gaumont and J.-C. Plaquevent, Tetrahedron Asymm., 14, 3081 (2003).

4. C.P. Mehnert, Chem. Eur. J., 11, 50 (2005)

5. S.-G. Lee, Y.J. Zhang, J.Y. Piao, H. Yoon, C.E. Song, J.H. Choi and J. Hong, Chem. Commun., 20, 2624 (2003).

6. C. Baleizão, B. Gigante, H. García and A. Corma, Tetrahedron, 60, 10461 (2004).

7. J.J. Jodry and K. Mikami, Tetrahedron Lett., 45, 4429 (2004).

8. J. H. Davis Jr., Chem. Lett., 33, 1072 (2004).

9. D.W. Kim and D.Y. Chi, Angew. Chem. Int. Ed. Engl., 43, 483 (2004).

10. I. Kawasaki, K. Tsunoda, T. Tsuji, T. Yamaguchi, H. Shibuta, N. Uchida, M. Yamashita and S. Ohta, Chem. Commun., 2134 (2005).

11. P. Walden, Bull. Acad. Imp. Sci., 1800 (1914).

12. P. Wasserscheid and W. Keim, Angew. Chem. Int. Ed., 39, 3772 (2000).

13. P. Wasserscheid and T. Welton, Ionic Liquids in Synthesis, Wiley-VCH Verlag: Weinheim, Germany (2003).
14. A.P. Abbott, G. Capper, D.L. Davies, H.L. Munro, R.K. Rasheed and V. Tambyrajah, Chem. Commun., 2010 (2001).

15. A.P. Abbott, G. Capper, D.L. Davies and R. Rasheed, Inorg. Chem., 43, 3447 (2004).

16. A.P. Abbott, G. Capper, D.L. Davies, R.K. Rasheed and V. Tambyrajah, Trans. Inst. Met. Finish., 79, 204 (2001).

17. A.P. Abbott, D. Boothby, G. Capper, D.L. Davies and R.K. Rasheed, J. Am. Chem. Soc., 126, 9142 (2004).

18. A.P. Abbott, G. Capper, D.L. Davies, R.K. Rasheed and V. Tambyrajah, Chem. Commun., 1, 70 (2003).

19. K. Othmer, Encyclopedia of Chemical Technology, Wiley, New York, Vol. 5, p. 476 (1993).

20. R.P. Swatloski, S.K. Spear, J.D. Holbrey and R.D. Rogers, J. Am. Chem. Soc., 124, 4974 (2002).

21. X. Li, B. Li, M. Cheng, Y. Du, X. Wang and P. Yang, J. Mol. Liq., 284, 1 (2008).

22. D.S. Zhao, H. Li, J. Zhang, L. Fu, M. Liu, J. Fu and P. Ren, Carbohydr. Polym., 87, 1490 (2012).

23. H.R. Liu, H.W. Yu, E.P. Zhou, X.H. Zhang and X.C. Zhang, Asian J. Chem., 25, 8266 (2013).

24. Z.B. Zhou, H. Matsumoto and K. Tatsumi, Chem. Eur. J., 10, 6581 (2004). 